\title{
Relación entre el rendimiento académico y el apoyo social de los profesores en estudiantes de educación superior en Angola
}

\section{Relationship between academic achievement and social support of lecturers in higher education students in Angola}

\author{
Liliana De Sousa*, Eva M ${ }^{\mathrm{a}}$ Taboada**, Rosa Ma Rivas**, Patricia Ma Iglesias** y Santiago López** \\ *Universidade Óscar Rivas (Angola), **Dpto. de Psicología Evolutiva y de la Educación. Universidade de Santiago de Compostela \\ (España)
}

\begin{abstract}
Resumen
Las investigaciones realizadas en el contexto universitario muestran la influencia del docente en la promoción del rendimiento académico a través de las relaciones que establecen con los estudiantes. El objetivo de este trabajo es analizar la relación entre el rendimiento académico y la percepción de los alumnos sobre el apoyo social proporcionado por sus profesores. La muestra estaba constituida por 598 estudiantes de Educación Superior de Angola. Se constata que la percepción de apoyo social proporcionado por los profesores aumenta con el incremento del rendimiento académico.

Palabras clave: Educación superior, rendimiento académico, apoyo social.
\end{abstract}

\begin{abstract}
Research conducted in the University context has shown the influence of the relationship teacher-student in attaining a higher academic achievement. The aim of this job was study is to analyze the relationship between academic achievement and student's perception of social support provided by their lecturers. The sample included 598 students of Higher Education in Angola. Student's perceptions of social support provided by their lecturers increases with increasing academic achievement.

Key words: Higher education, academic achievement, social support.
\end{abstract}

Los profesores son actores fundamentales de las instituciones de Enseñanza Superior. Como docentes, orientan, facilitan e incentivan el proceso de enseñanza-aprendizaje y fomentan que el alumnado valore y actualice continuamente sus conocimientos (Masseto, 2005). A su vez, gestionan las interacciones dentro del aula y, a menudo, se convierten en modelos a seguir por los estudiantes.

Aunque las características de la Educación Superior (grupos más grandes, mayor anonimato) fomentan una relación profesor-alumno más impersonal que en etapas anteriores, y menores posibilidades de que los estudiantes se beneficien del apoyo socio-afectivo del profesor (Rego, 1998), la importancia de la dimensión afectiva en la relación pedagógica ha sido destacada por distintos autores (Zabalza, 2003), y algunos estudios han puesto de manifiesto el papel de los profesores en la promoción del éxito académico (Formiga, 2007).

Garbanzo (2007) sostiene al respecto la importancia de las expectativas creadas por los estudiantes en sus relaciones con profesores y compañeros de clase, y Castejón y Pérez (1998) defienden que el alumnado espera encontrar en el profesor una relación emocional que tendrá impacto sobre su rendimiento.

De forma más específica, Malecki y Demaray (2005) comprobaron que el apoyo emocional de los profesores era un predictor significativo de las habilidades sociales y de la competencia académica.

Así, el apoyo social proporcionado tanto por familiares y amigos como por profesores, ha demostrado tener una influencia relevante en la adaptación a la educación superior. Si bien, la relación entre estas variables está mediada por otras como el género, dado que los hombres puntúan más alto que las mujeres en dimensiones como la percepción de competencia, el desarrollo de la carrera, el bienestar psicológico o la relación con compañeros y profesores (Pinheiro, 2003).

No obstante, y a pesar de que el apoyo de los profesores parece favorecer el desarrollo de sentimientos positivos y el refuerzo de atribuciones causales positivas que aumentan la confianza, la autoestima, la motivación $\mathrm{y}$, consecuentemente, el rendimiento académico; las investigaciones en el ámbito universitario no son abundantes.

Por ello, el objetivo del presente trabajo es determinar la relación entre el apoyo social de los profesores percibido por estudiantes de Educación Superior en Angola.

\section{Método}

\section{Participantes}

La muestra estaba formada por 598 estudiantes universitarios angoleños de segundo $(n=368)$ y cuarto curso $(\mathrm{n}=230)$ con edades comprendidas entre los $18 \mathrm{y}$ los 59 años ( $\bar{X}=26.5, S D=7.0)$, de los cuales $346(57.9 \%)$ eran hombres y $252(42.1 \%)$ mujeres. La mayoría de los 
participantes (72.2\%) estaban solteros y el $45.1 \%$ compaginaba estudios y trabajo. El $60.0 \%$ de estos contaba con el estatuto de estudiante- trabajador.

Tres cuartas partes $(74.6 \%)$ realizaban estudios del área de ciencias sociales y jurídicas, el $17.7 \%$ de ciencias de la salud y el $7.7 \%$ restante de ingenierías.

En relación a las características sociofamiliares y de convivencia, en conjunto, los padres de los participantes, poseían estudios de nivel medio (36.0\%) o superiores $(33.6 \%)$, si bien, el porcentaje de progenitores con estudios básicos era mayor entre las madres que entre los padres (19.1\% vs $10.9 \%)$, tendencia que se invertía en relación a los estudios superiores (padres: 40.0\%; madres: $27.3 \%$ ). Tres cuartas partes vivía en unidades familiares formadas por entre 3 y 8 miembros, la mitad $(51.0 \%)$ lo hacía en régimen de vivienda propia y la mayoría (64.2\%) se mostraba bastante o muy satisfecho con la misma.

\section{Instrumentos}

La percepción del apoyo social proporcionado por los profesores se evaluó a través de un Cuestionario elaborado ad hoc, formado por siete ítems, con una escala de respuesta tipo Likert de 1 a 5 puntos, oscilando desde totalmente en desacuerdo a totalmente de acuerdo. Los ítems se referían a la percepción de apoyo, confianza y preocupación por parte de los profesores, así como a la cercanía que mostraban los mismos.

Los datos sobre el rendimiento académico se obtuvieron a través de la nota media del último semestre.

\section{Análisis}

El análisis de datos se llevó a cabo a través del programa IBM SPSS Statistics, versión 20.

Se analizó la relación entre el apoyo social percibido de los profesores y las variables sexo, estado civil, curso académico $\mathrm{y}$ área de formación, mediante las correspondientes pruebas para el contraste de medias ( $t$ de Student y ANOVA).

Se calculó el coeficiente de correlación de Pearson entre el rendimiento académico y el apoyo social percibido, y se realizó un ANOVA de un factor para determinar si existían diferencias en el nivel de apoyo en función del rendimiento académico categorizado en tres niveles (bajo, medio y alto).

\section{Resultados}

Los resultados indican que la percepción por parte de los estudiantes del apoyo de sus profesores es similar en ambos sexos (ver Tabla 1).

Tabla 1.

Apoyo social percibido de los profesores en función del sexo

\begin{tabular}{cccccr}
\hline & \multicolumn{3}{c}{ Hombres } & \multicolumn{3}{c}{ Mujeres } \\
\cline { 2 - 6 } Apoyo social & $\square$ & $S D$ & $\square$ & $S D$ & $t$ \\
\cline { 2 - 6 } & 3.4 & 0.6 & 3.4 & 0.6 & -3.51 \\
\hline
\end{tabular}

Tampoco se obtienen diferencias significativas en relación al estado civil (ver Tabla 2) o al curso académico (ver Tabla 3).

Tabla 2.

Apoyo social percibido de los profesores en función del estado civil

\begin{tabular}{lccccccc}
\hline & \multicolumn{3}{c}{ Soltero } & \multicolumn{5}{c}{ Casado } & \multicolumn{3}{c}{$\begin{array}{c}\text { Pareja de } \\
\text { hecho }\end{array}$} \\
\cline { 2 - 8 } & \multicolumn{1}{c}{ A } & $S D$ & $\bar{x}$ & $S D$ & $\bar{x}$ & $S D$ & $F$ \\
\cline { 2 - 8 } $\begin{array}{l}\text { Apoyo } \\
\text { social }\end{array}$ & 3.4 & 0.6 & 3.4 & 0.5 & 3.4 & 0.7 & 0.165 \\
\hline
\end{tabular}

Tabla 3.

Apoyo social percibido de los profesores en función del curso

\begin{tabular}{cccccc}
\hline & \multicolumn{2}{c}{ Segundo } & \multicolumn{3}{c}{ Cuarto } \\
\cline { 2 - 6 } Apoyo social & $\bar{x}$ & $S D$ & $\bar{x}$ & $S D$ & $t$ \\
\cline { 2 - 6 } & 3.4 & 0.6 & 3.4 & 0.6 & -1.152 \\
\hline
\end{tabular}

Por el contrario, si se analiza la variable edad, la correlación es estadísticamente significativa $(p=.048)$ y positiva, aunque débil, en relación a la percepción de los estudiantes respecto al apoyo de sus profesores. Datos similares se obtienen al considerar el área de formación. La Tabla 4 muestra una diferencia marginalmente significativa, $F(2,515)=2.993, p=0.51$, siendo los estudiantes de los cursos de ingenierías los que perciben un mayor soporte por parte de los profesores.

Tabla 4.

Apoyo social percibido de los profesores en función del área de formación

\begin{tabular}{|c|c|c|c|c|c|c|c|}
\hline & \multicolumn{2}{|c|}{$\begin{array}{c}\text { Ciencias } \\
\text { de la } \\
\text { salud } \\
\end{array}$} & \multicolumn{2}{|c|}{$\begin{array}{l}\text { Ciencias } \\
\text { sociales }\end{array}$} & \multicolumn{3}{|c|}{ Ingenierías } \\
\hline & $\bar{x}$ & SD & $\bar{x}$ & SD & $\bar{x}$ & SD & $F$ \\
\hline $\begin{array}{l}\text { Apoyo } \\
\text { social }\end{array}$ & 3.4 & 0.7 & 3.2 & 0.8 & 3.4 & 0.6 & $2.993^{*}$ \\
\hline
\end{tabular}

Al valorar en su conjunto a los estudiantes que acceden a la Educación Superior en Angola, los resultados indican que el valor medio de la percepción del apoyo de los profesores aumenta conforme lo hace el rendimiento académico, pero sin que las diferencias entre los tres niveles del mismo sean significativas (ver Tabla 5).

Tabla 5.

Apoyo social percibido de los profesores en función del rendimiento académico

\begin{tabular}{lrrrrrrc}
\hline & \multicolumn{3}{c}{ R. Bajo } & \multicolumn{2}{c}{ R. Medio } & \multicolumn{2}{c}{ R. Alto } \\
\cline { 2 - 8 } & $\bar{x}$ & $S D$ & $\bar{x}$ & $S D$ & $\bar{x}$ & $S D$ & $F$ \\
\cline { 2 - 8 } $\begin{array}{l}\text { Apoyo } \\
\text { social }\end{array}$ & 22.8 & 3.9 & 23.9 & 4.5 & 24.3 & 4.2 & 2.195 \\
\hline
\end{tabular}

No obstante, a pesar de resultar significativo $(p \leq .05)$, hay que tener en cuenta que el valor obtenido para el coeficiente de correlación de Pearson entre ambas variables fue de .099 . 


\section{Discusión}

Investigaciones realizadas tanto en educación secundaria como en Educación Superior muestran que el apoyo percibido por los profesores fomenta la participación de los estudiantes en las actividades de aprendizaje (Dupont, Galand, Hospel \& Nils, 2014) y que se asocia con la motivación de los alumnos, lo que, a su vez, conlleva un mayor compromiso a nivel comportamental, cognitivo y emocional (Dupont, Galand \& Nils, 2015).

No obstante, este resultado no es consistente a lo largo de la literatura, ya que otros estudios realizados con muestras universitarias no encontraron relaciones significativas entre el rendimiento académico y el apoyo social percibido por parte de profesores, padres, compañeros de clase y amigos cercanos/parejas (D’Alessio, Lavagnino, Lupano, Ongarato y Becerra, 2008).

La percepción del apoyo social por parte de los profesores no parece ser un factor determinante para el rendimiento de los estudiantes universitarios en Angola.

Alarcão (2000) señala que el fracaso escolar en los estudiantes universitarios es un problema multidimensional que puede estar asociado, además de a otros factores, con los profesores. Características como la implicación en la relación con los alumnos, la competencia pedagógica, la dispersión por exceso de actividades, o la incapacidad para identificar las dificultades de sus estudiantes, entre otros, influyen en el rendimiento. Estas mismas características pueden conllevar un menor acercamiento entre estudiantes y docentes.

En conclusión, sería necesario tener en cuenta las características de la sociedad, de las personas que acceden a la Educación Superior, y especialmente, el perfil y las condiciones laborales de los docentes.

\section{Referencias}

Alarcão, I. (2000). Para uma conceptualização dos fenómenos de insucesso/sucesso escolares no ensino superior. En J. Tavares \& R. Santiago (Eds.). Ensino Superior: (in) sucesso académico (13-23). Porto: Porto Editora.

Castejón, C. y Pérez, S. (1998). Un modelo causal-explicativo sobre la influencia de las variables psicosociales en el rendimiento académico. Revista Bordon. Sociedad Española de Pedagogía, 2(50), 170-184.

D’Alessio, A. Lavagnino, A., Lupano, M. Ongarato, P. \& Becerra, L. (Agosto, 2008). Apoyo social percibido y rendimiento académico en estudiantes universitarios. Trabajo presentado en las XV Jornadas de Investigación y Cuarto Encuentro de Investigadores en Psicología del Mercosur. Buenos Aires, 2008.

Dupont, S., Galand, B., Hospel, V., \& Nils, F. (2014). Social context, self-perceptionsand student engagement: A SEM investigation of the self-system model of moti-vational development. Electronic Journal of Research in Educational Psychology, 12, 532. http://dx.doi.org/10.14204/ejrep.32.13081
Dupon, S., Galand, B. \& Nils, F. (2015). The impact of different sources of social support on academic performance: Intervening factors and mediated pathways in the case of master's thesis. Revue européenne de psychologie appliquée, 65, 227-237. http://dx.doi.org/10.1016/j.erap.2015.08.003

Formiga, N. (2007). Pais e professores como explicação de variáveis do rendimento escolar. Universidade Federal da Paraíba. Recuperado de http://www.psicologia.com.pt/artigos/ver_artigo.php? codigo $=\mathrm{A} 0342$ \&area $=\mathrm{d} 6$

Garbanzo, C.M. (2007). Factores asociados al rendimiento académico en estudiantes universitarios. Una reflexión desde la calidad educativa de la educación superior pública. Revista Educación, 31(1), 43-63. http://dx.doi.org/10.15517/revedu.v31i1.1252

Malecki, C. K., \& Demaray, M. K. (2006). Social support as a buffer in the relationship between socioeconomic status and academic performance. School Psychology Quarterly, 21), 375-395. http://dx.doi.org/10.1037/h0084129

Masetto, M. (2005). Docência Universitária: repensando a aula. En A. Teodoro y M.L. Vasconcelos (Orgs.). Ensinar e aprender no ensino superior: por uma epistemologia da curiosidade na formação universitária, (2 ${ }^{\mathrm{a}}$ Ed.) (pp.79-108). São Paulo: Cortez/Mackenzie.

Pinheiro, M. (2003). Uma época especial: Suporte social e vivências académicas na transição e adaptação ao ensino superior (Tesis doctoral). Universidade de Coimbra, Coimbra.

Rego, T. C. (1998). Educação, Cultura e Desenvolvimento: o que pensam os professores sobre as diferenças individuais. En J.G. Aquino (Org.). Diferenças e preconceitos na escola: alternativas teóricas e práticas (49-71). São Paulo: Summus Editorial.

Zabalza, M. (2003). Competências docentes del profesorado universitario. Calidad y desarrollo profesional. Madrid: Narcea. 